\title{
Candidate One-Way Functions Based on Expander Graphs
}

\author{
Oded Goldreich
}

\begin{abstract}
We suggest a candidate one-way function using combinatorial constructs such as expander graphs. These graphs are used to determine a sequence of small overlapping subsets of input bits, to which a hard-wired random predicate is applied. Thus, the function is extremely easy to evaluate: All that is needed is to take multiple projections of the input bits, and to use these as entries to a look-up table. It is feasible for the adversary to scan the look-up table, but we believe it would be infeasible to find an input that fits a given sequence of values obtained for these overlapping projections.

The conjectured difficulty of inverting the suggested function does not seem to follow from any well-known assumption. Instead, we propose the study of the complexity of inverting this function as an interesting open problem, with the hope that further research will provide evidence to our belief that the inversion task is intractable.
\end{abstract}

Keywords: One-Way Functions, Expander Graphs.

This work appeared as TR00-090 of ECCC. The current revision is intentionally minimal, because the original publication has triggered several subsequent works (although less than we have hoped). For further discussion of these subsequent works and some afterthoughts, see Section 6 .

\section{Introduction}

In contrary to the present attempts to suggest a practical private-key encryption scheme to replace the DES, we believe that attempts should focus on suggesting practical one-way functions and pseudorandom functions. Being a simpler object, one-way functions should be easier to construct, and such constructions may later yield directly or indirectly a variety of other applications (including private-key encryption schemes).

The current attempts to suggest a practical private-key encryption scheme in place of the DES seem quite ad-hoc: Not only that they cannot be reduced to any well-known problem, but (typically) they do not relate to a computational problem of natural appeal. Thus, the study of these suggestions is of limited appeal (especially from a conceptual point of view).

In this manuscript, we propose a general scheme for constructing one-way functions. We do not believe that the complexity of inverting the resulting function follows from some well-known intractability assumptions. We believe that 
the complexity of inverting this function is a new interesting open problem, and hope that other researcher will be able to obtain better understanding of this problem.

In addition to the abstract presentation, we propose several concrete instantiations of our proposal. It seems to us that a reasonable level of "security" (i.e., hardness to invert) may be achieved at very modest input lengths. Specifically, on input length at the order of a couple of hundreds of bits, inverting the function may require complexity (e.g., time) beyond $2^{100}$.

Style and Organization. This write-up is intended to two different types of readers, since we believe that it is relevant to two different research communities (i.e., computational complexity and applied cryptography). Consequently, we provide an asymptotic presentation as well as suggestions for concrete parameters. The basic suggestion is presented in Sections 2 and 3. Concrete instantiations of this suggestion are proposed in Section 4. Concluding comments appear in Section 5.

\section{The Basic Suggestion}

We construct a (uniform) collection of functions $\left\{f_{n}:\{0,1\}^{n} \rightarrow\{0,1\}^{n}\right\}_{n \in \mathbb{N}}$. Our construction utilizes a collection of $\ell(n)$-subsets, $S_{1}, \ldots, S_{n} \subset[n] \stackrel{\text { def }}{=}\{1, \ldots, n\}$, and a predicate $P:\{0,1\}^{\ell(n)} \rightarrow\{0,1\}$. Jumping ahead, we hint that:

1. The function $\ell$ is relatively small: Theoretically speaking, $\ell=O(\log n)$ or even $\ell=O(1)$. In practice $\ell$ should be in the range $\{7, \ldots, 16\}$, whereas $n$ should range between a couple of hundreds and a couple of thousands.

2. We prefer to have $P:\{0,1\}^{\ell} \rightarrow\{0,1\}$ be a random predicate. That is, it will be randomly selected, fixed, and "hard-wired" into the function. For sure, $P$ should not be linear, nor depend on few of its bit locations.

3. The collection $S_{1}, \ldots, S_{n}$ should be expanding; specifically, for some $k$, every $k$ subsets should cover at least $k+\Omega(n)$ elements of $\{1, \ldots, n\}$. The complexity of the inversion problem (for $f_{n}$ constructed based on such a collection) seems to be exponential in the "net expansion" of the collection (i.e., the cardinality of the union minus the number of subsets).

For $x=x_{1} \cdots x_{n} \in\{0,1\}^{n}$ and $S \subset[n]$, where $S=\left\{i_{1}, i_{2}, \ldots, i_{t}\right\}$ and $i_{j}<i_{j+1}$, we denote by $x_{S}$ the projection of $x$ on $S$; that is, $x_{S}=x_{i_{1}} x_{i_{2}} \cdots x_{i_{t}}$. Fixing $P$ and $S_{1}, \ldots, S_{n}$ as above, we define

$$
f_{n}(x) \stackrel{\text { def }}{=} P\left(x_{S_{1}}\right) P\left(x_{S_{2}}\right) \cdots P\left(x_{S_{n}}\right) .
$$

Note that we think of $\ell$ as being relatively small (i.e., $\ell=O(\log n)$ ), and aim at having $f_{n}$ be univertible within time $2^{n / O(1)}$. Thus, the hardness of inverting $f_{n}$ cannot be due to the hardness of inverting $P$. Instead, the hardness of inverting $f_{n}$ is supposed to come from the combinatorial properties of the collection of sets $C=\left\{S_{1}, \ldots, S_{n}\right\}$ (as well as from the combinatorial properties of predicate $P)$. 


\subsection{The preferred implementation}

Our preference is to have $P$ be a fixed randomly chosen predicate, which is hard-wired into the algorithm for evaluating $f_{n}$. Actually, one better avoid some choices; see next section. (In case $\ell=\Theta(\log n)$ bad choices are rare enough.) In practice, we think of $\ell$ in the range $\{7, \ldots, 16\}$, and so hard-wiring a (random) predicate defined on $\{0,1\}^{\ell}$ is quite feasible. The $\ell$-subsets will be determined by combinatorial constructions called expander graphs. At this point the reader may think of them too as being hard-wired into the algorithm. On input $x \in\{0,1\}^{n}$, the algorithm for computing $f_{n}$ proceeds as follows:

1. For $i=1, . ., n$, projects $x$ on $S_{i}$, forming the $\ell$-bit long string $x^{(i)}$.

2. For $i=1, . ., n$, by accessing a look-up table for $P$, determines the bit $y_{i}=$ $P\left(x^{(i)}\right)$.

The output is the $n$-bit long string $y_{1} y_{2} \cdots y_{n}$.

(Note that the $n$ operations, in each of the foregoing two steps, can be performed in parallel.)

\subsection{An alternative implementation}

An alternative to having $P$ "hard-wired" to the algorithm (as above) is to have it appear as part of the input (and output). That is, letting $\langle P\rangle$ denote the $2^{\ell}$-bit string that fully specifies $P$, we have

$$
f_{n}^{\prime}(\langle P\rangle, x) \stackrel{\text { def }}{=}\left(\langle P\rangle, P\left(x_{S_{1}}\right) P\left(x_{S_{2}}\right) \cdots P\left(x_{S_{n}}\right)\right)
$$

Thus, $P$ is essentially random, since the inversion problem is considered with respect to a uniformly chosen input. This implementation is more appealing from a theoretical point of view, and in such a case one better let $\ell=\log _{2} n$ (rather than $\ell=O(1))^{1}$

\subsection{Determining suitable collections}

As hinted above, the collection of $\ell$-subsets, $C=\left\{S_{1}, \ldots, S_{n}\right\}$, is to be determined by a suitable combinatorial construction known as expander graphs. The reason for this choice will become more clear from the analysis of one obvious attack (presented in Section 3.2). The specific correspondence (between expanders and subsets) depends on whether one uses the bipartite or non-bipartite formulation of expander graphs:

Bipartite formulation: In this case one considers a bipartite graph $B=((U, V), E)$, where $(U, V)$ is a partition of the vertex set, with $|U|=|V|$, and $E \subset U \times V$ is typically sparse. The expanding property of the graph provides, for every

\footnotetext{
${ }^{1}$ Our main concern at the time was to make the new assumption as strong as possble. From that perspective, we preferred $\ell=O(\log n)$ over $\ell=O(1)$.
} 
$U^{\prime} \subset U$ (of size at most $|U| / 2$ ), a lower bound on $\left|\Gamma\left(U^{\prime}\right)\right|-\left|U^{\prime}\right|$ (in terms of $\left.\left|U^{\prime}\right|\right)$, where $\Gamma\left(U^{\prime}\right)=\left\{v: \exists u \in U^{\prime}\right.$ s.t. $\left.(u, v) \in E\right\}$.

Our collection of subsets will be defined as $C=\left\{S_{u}\right\}_{u \in U}$, where $S_{u}=\{v$ : $(u, v) \in E\}$.

Non-bipartite formulation: In this case one considers a graph $G=(V, E)$, so that for every $V^{\prime} \subset V$ (of size at most $|V| / 2$ ), a suitable lower bound on $\left|\Gamma\left(V^{\prime}\right) \backslash V^{\prime}\right|$ holds, where $\Gamma\left(V^{\prime}\right)=\left\{v: \exists v^{\prime} \in V^{\prime}\right.$ s.t. $\left.\left(v^{\prime}, v\right) \in E\right\}$.

Our collection of subsets is defined as $C=\left\{S_{v}\right\}_{v \in V}$, where $S_{v}=\{w$ : $(v, w) \in E\} \cup\{v\}$.

In both cases, the lower bound provided by the expansion property on the size of the neighbor set is linear in the size of the specific vertex set; e.g., for the non-bipartite formulation it holds that $\left|\Gamma\left(V^{\prime}\right) \backslash V^{\prime}\right| \geq c \cdot\left|V^{\prime}\right|$ for some constant $c>0$ and every admissible $V^{\prime}$.

\section{$3 \quad$ Avoiding obvious weaknesses}

Considering a few obvious attacks, we rule out some obviously bad choices of the predicate $P$ and the collection $C$.

\subsection{The choice of the predicate}

We start by discussing two bad choices (for the predicate $P$ ), which should be avoided.

Linear predicates. It is certainly bad to use a linear predicate $P$ (i.e., $P\left(\sigma_{1} \cdots \sigma_{\ell}\right)=$ $p_{0}+\sum_{i=1}^{\ell} p_{i} \sigma_{i}$, for some $\left.p_{0}, p_{1}, \ldots, p_{\ell} \in\{0,1\}\right)$. Under a linear $P$, the question of inverting $f_{n}$, regardless of what collection of subsets $C$ is used, boils down to solving a linear system (of $n$ equations in $n$ variables), which is easy. Having a predicate $P$ that is close to a linear predicate is dangerous too.

Horn predicates. Likewise, one should avoid having any predicate that will make the system of equations (or conditions) solvable in polynomial-time. The only other type of easily solvable equations are these arising from Horn formulae (e.g., an OR of all variables).

Degenerate predicates. The rest of our analysis refers to the collection of sets that determine the inputs to which the predicate $P$ is applied. For this analysis to be meaningful, the predicate should actually depend on all bits in its input (i.e., be non-degenerated).

Good predicates. We believe that most predicates are good for our purpose. In particular, we suggest to use a uniformly chosen predicate. 


\subsection{The choice of the collection}

Since the inverting algorithm can afford to consider all preimages of the predicate $P$, it is crucial that the inversion of $f_{n}$ cannot be performed by interactively inverting $P$. To demonstrate this point, consider the case $\ell=1$ and the collection $\left\{S_{1}, \ldots, S_{n}\right\}$ such that $S_{i}=\{i\}$. In this case the $S_{i}$ 's are disjoint and we can recover the preimage by inverting $P$ on each of the bits of the image, separately from the others. For a less trivial example, consider the case where the collection $C$ consists of $n / 2 \ell$ sub-collections, each having $2 \ell$ subsets of some distinct set of $2 \ell$ elements. In this case, inversion can be performed in time $O\left(n \cdot 2^{2 \ell}\right)$ by considering each of these disjoint sets (of $2 \ell$ elements) separately. Recall that we wish the complexity of inversion to be exponential in $n$ (and not in $\ell$, which may be a constant).

In general, a natural inverting algorithm that should be defeated is the following: On input $y=f_{n}(x)$, the algorithm proceeds in $n$ steps, maintaining a list of partially specified preimages of $y$ under $f_{n}$. Initially, the list consists of the unique fully-undetermined string $*^{n}$. In the first step, depending on the first bit of $y=y_{1} \cdots y_{n}$, we form the list $L_{1}$ of strings over $\{*, 0,1\}$ such that, for every $z \in L_{1}$, it holds that $P\left(z_{S_{1}}\right)=y_{1}$ and $z_{[n] \backslash S_{1}}=*^{n-\ell}$, where $[m] \stackrel{\text { def }}{=}\{1, \ldots, m\}$ (and $z_{S_{1}} \in\{0,1\}^{\ell}$ ). In the $i+1^{\text {st }}$ step, we extend $L_{i}$ to $L_{i+1}$ in the natural manner:

- Let $U^{\prime}=\cup_{j=1}^{i} S_{j}$ and $U=\cup_{j=1}^{i+1} S_{j}$.

- For every $z^{\prime} \in L_{i}$, we consider all $2^{\left|U \backslash U^{\prime}\right|}$ strings $z \in\{*, 0,1\}^{n}$ satisfying

1. $z_{U^{\prime}}=z_{U^{\prime}}^{\prime}$

2. $z_{U \backslash U^{\prime}} \in\{0,1\}^{\left|U \backslash U^{\prime}\right|}$, and

3. $z_{[n] \backslash U}=*^{n-|U|}$.

The string $z$ is added to $L_{i+1}$ if and only if $P\left(z_{S_{i+1}}\right)=y_{i+1}$.

Thus (when invoked on input $y$ ), for every $i$, it holds that

$$
L_{i}=\left\{z \in\{*, 0,1\}^{n}: \begin{array}{l}
z_{k}=* \text { if and only if } k \in[n] \backslash \cup_{j=1}^{i} S_{j} \\
\\
P\left(z_{S_{j}}\right)=y_{j} \text { for } j=1, \ldots, i
\end{array}\right\} .
$$

The average running-time of this algorithm is determined by the expected size of the list at step $i$, for the worst possible $i$. Letting $U=\cup_{j=1}^{i} S_{j}$, consider the set

$$
A_{\sigma_{1} \cdots \sigma_{i}} \stackrel{\text { def }}{=}\left\{z \in\{*, 0,1\}^{n}: \begin{array}{l}
z_{k}=* \text { if and only if } k \in[n] \backslash U \\
\\
P\left(z_{S_{j}}\right)=\sigma_{j} \text { for } j=1, \ldots, i
\end{array}\right\},
$$

and let $X$ be uniformly distributed over $\{0,1\}^{n}$. Then, the expected size of $L_{i}$ equals

$$
\sum_{\alpha \in\{0,1\}^{i}} \operatorname{Pr}\left[f(X)_{[i]}=\alpha\right] \cdot\left|A_{\alpha}\right|=\sum_{\alpha \in\{0,1\}^{i}} \operatorname{Pr}\left[\exists z \in A_{\alpha} \text { s.t. } X_{U}=z_{U}\right] \cdot\left|A_{\alpha}\right|
$$




$$
\begin{aligned}
& =\sum_{\alpha \in\{0,1\}^{i}} \frac{\left|A_{\alpha}\right|}{2^{|U|}} \cdot\left|A_{\alpha}\right|=2^{-|U|} \cdot \sum_{\alpha \in\{0,1\}^{i}}\left|A_{\alpha}\right|^{2} \\
& \geq 2^{-|U|} \cdot \frac{\left(2^{|U|}\right)^{2}}{2^{i}}=2^{|U|-i}
\end{aligned}
$$

where the inequality is due to the fact that the minimum value of $\sum_{i} z_{i}^{2}$, taken over $M\left(=2^{i}\right)$ non-negative $z_{i}$ 's summing to $N\left(=2^{|U|}\right)$, is obtained when the $z_{i}$ 's are equal, and the value itself is $M \cdot(N / M)^{2}=N^{2} / M$.

Note that the algorithm needs not proceed by the foregoing order of sets (i.e., $\left.S_{1}, S_{2}, S_{3}, \ldots\right)$. In general, for every 1 -1 permutation $\pi$ over $[n]$, we may proceed by considering in the $i^{\text {th }}$ step the set $S_{\pi(i)}$. Still, the complexity of this (generalized) algorithm is at least exponential in

$$
\min _{\pi}\left\{\max _{i}\left\{\left|\cup_{j=1}^{i} S_{\pi(j)}\right|-i\right\}\right\} \text {. }
$$

We should thus use a collection such that Eq. (3) is big (i.e., bounded below by $\Omega(n))$.

Bad collections. Obviously, it is a bad idea to have $S_{j}=\{j+1, \ldots, j+\ell\}$, since in this case we have $\left|\cup_{j=1}^{i} S_{j}\right|-i \leq \ell-1$ for every $i$. It also follows that we cannot use $\ell \leq 2$, since in this case one can always find an order $\pi$ such that Eq. (3) is bounded above by $\ell-1$.

Good collections. An obvious lower bound on Eq. (3) is obtained by the expansion property of the collection $C=\left\{S_{j}\right\}$, where the expansion of $C$ is defined as

$$
\max _{k} \min _{I:|I|=k}\left\{\left|\cup_{j \in I} S_{j}\right|-k \mid\right\} \text {. }
$$

A natural suggestion is to determine the collection $C$ according to the neighborhood sets of an expander graph. Loosely speaking, known constructions of expander graphs allow to let $\ell$ be a small constant (in the range $\{7, \ldots, 16\}$ ), while guaranteeing that Eq. (4) is a constant fraction of $n$.

\section{Concrete parameters for practical use}

If we go for random predicates, then we should keep $\ell$ relatively small (say, $\ell \leq 16)$, since our implementation of the function must contain a $2^{\ell}$-size table look-up for $P$. (Indeed, $\ell=8$ poses no difficulty, and $\ell=16$ requires a table of $64 \mathrm{~K}$ bits which seems reasonable.) For concrete security we will be satisfied with time complexities such as $2^{80}$ or so. Our aim is to have $n$ as small as possible (e.g., a couple of hundreds).

The issue addressed below is which expander to use. It is somewhat "disappointing" that for some specific parameters we aim for, we cannot use the "best" known explicit constructions.

Below we use the bipartite formulation of expanders. By expansion we mean a lower bound established on the quantity in Eq. (4). Recall that the time complexity is conjectured to be exponential in this bound. 
Random construction. This yields the best results, but the "cost" is that with small probability we may select a bad construction. (The fact that we need to hard-wire the construction into the function description is of little practical concern, since we are merely talking of hard-wiring $n \cdot \ell \cdot \log _{2} n$ bits, which for the biggest $n$ and $\ell$ considered below merely means hard-wiring $20 \mathrm{~K}$ bits.) Alternatively, one may incorporate the specification of the construction in the input of the one-way function, at the cost of augmenting the input by $n \cdot \ell \cdot \log _{2} n$ (where the original input is $n$-bit long). Specific values that may be used are tabulated in Figure 1. ${ }^{2}$

\begin{tabular}{|r|r|r|r|}
\hline degree (i.e., $\ell$ ) & \#vertices (i.e., $n$ ) & expansion & error prob. \\
\hline \hline 10 & 256 & 77 & $2^{-81}$ \\
\hline 12 & 256 & 90 & $2^{-101}$ \\
\hline 14 & 256 & 103 & $2^{-104}$ \\
\hline 16 & 256 & 105 & $2^{-152}$ \\
\hline \hline 8 & 384 & 93 & $2^{-83}$ \\
\hline 10 & 384 & 116 & $2^{-121}$ \\
\hline 12 & 384 & 139 & $2^{-141}$ \\
\hline \hline 8 & 512 & 130 & $2^{-101}$ \\
\hline 10 & 512 & 159 & $2^{-151}$ \\
\hline 12 & 512 & 180 & $2^{-202}$ \\
\hline
\end{tabular}

Fig. 1. The parameters of a random construction

The last column (i.e., error prob.) states the probability that a random construction (with given $n$ and $\ell$ ) does not achieve the stated expansion. Actually, we only provide upper bounds on these probabilities.

Alon's Geometric Expanders [4]. These constructions do not allow $\ell=$ $O(\log n)$, but rather $\ell$ that is polynomially related to $n$. Still for our small numbers we get meaningful results, when using $\ell=q+1$ and $n=q^{2}+q+1$, where $q$ is a prime power. Specific values that may be used are tabulated in Figure $2 .^{3}$ Note that these are all the suitable values for Alon's construction (with $\ell \leq 16$ ); in particular, $\ell$ uniquely determines $n$ and $\ell-1$ must be a prime power.

2 The expansion was computed in a straightforward manner. The key issue is to provide, for any fixed $k$ and $h$, a good upper bound on the probability that a specific set of $k$ vertices has less than $h$ neighbors.

3 The expansion is computed from the eigenvalues, as in [5]. Actually, we use the stronger bound provided by [4, Thm. 2.3] rather than the simpler (and better known) bound. Specifically, the lower bounds in [4, Thm. 2.3] are on the size of the neighborhood of $x$-subsets, and so we should subtract $x$ from them, and maximize over all possible $x$ 's. (We use the stronger lower bound of $n-\frac{(n-x)(\ell n+1)}{\ell n+1+(n-\ell-2) x}$, rather than the simpler bound of $n-\frac{n^{3 / 2}}{x}$, both provided in [4, Thm. 2.3].) 


\begin{tabular}{|r|r|r|l|}
\hline degree (i.e., $\ell$ ) & \#vertices (i.e., $n$ ) & expansion & comment \\
\hline \hline 10 & 91 & 49 & expansion too low \\
\hline \hline 12 & 133 & 76 & quite good \\
\hline 14 & 183 & 109 & very good \\
\hline
\end{tabular}

Fig. 2. The parameters of Geometric expanders

The Ramanujan Expanders of Lubotzky, Phillips, and Sarnak [10]. Until one plays with the parameters governing this construction, one may not realize how annoying these may be with respect to an actual use: The difficulty is that there are severe restrictions regarding the degree and the number of vertices, ${ }^{4}$ making $n \approx 2000$ the smallest suitable choice. Admissible values are tabulated in Figure 3. ${ }^{5}$

\begin{tabular}{|r|r|c|r|r|rc|}
\hline \multicolumn{3}{|c|}{ Parameters } & \multicolumn{4}{c|}{ Results } \\
\hline$p$ & $q$ & bipartite? & $\ell$ & $n$ & expansion (+ comment) \\
\hline \hline 13 & 5 & NO & 15 & 120 & 20 & (unacceptable) \\
\hline \hline 5 & 13 & NO & 7 & 2184 & 160 & (better than needed) \\
\hline 13 & 17 & YES & 14 & 2448 & 392 & (better than needed) \\
\hline
\end{tabular}

Fig. 3. The parameters of Ramanujan expanders

Note that $p=5$ and $p=13$ are the only admissible choices for $\ell \leq 16$. Larger values of $q$ may be used, but this will only yield larger value of $n$.

Using the simple expander of Gaber-Galil [8]. Another nasty surprise is that the easy-to-handle expander of Gaber-Galil performs very poorly on our range of parameters. This expander has degree 7 (i.e., $\ell=7$ ), and can be constructed for any $n=m^{2}$, where $m$ is an integer. But its expansion is $(c / 2) \cdot n$, where $c=1-\sqrt{3 / 4} \approx 0.1339746$, and so to achieve expansion above 80 we need to use $n=1225$. See Figure 4 .

A second thought. In some applications having $n$ on the magnitude of a couple of thousands may be acceptable. In such a case, the explicit constructions of Lubotzky, Phillips, and Sarnak [10] and of Gaber and Galil [8] become relevant. In view of the lower degree and greater flexibility, we would prefer the construction of Gaber-Galil.

\footnotetext{
${ }^{4}$ Specifically, $\ell=p+1$ and $n=\left(q^{3}-q\right) / 2$, where $p$ and $q$ are different primes, both congruent to $1 \bmod 4$, and $p$ is a square $\bmod q$. For the non-bipartite case, $p$ is a non-square $\bmod q$, and $n=q^{3}-q$. Recall that for non-bipartite graphs $\ell$ equals the degree plus 1 (rather than the degree).

${ }^{5}$ The expansion is computed from the eigenvalues, as in [5].
} 


\begin{tabular}{|r|r|r|l|}
\hline degree (i.e., $\ell$ ) & \#vertices (i.e., $n$ ) & expansion & comment \\
\hline \hline 7 & 400 & 27 & expansion way too low \\
\hline \hline 7 & 1225 & 83 & good \\
\hline 7 & 1600 & 108 & very good \\
\hline 7 & 2500 & 168 & beyond our requirements \\
\hline
\end{tabular}

Fig. 4. The parameters of Gaber-Galil expanders

\section{Concluding remarks}

This was the last section of the original write-up.

\subsection{Variations}

One variation is to use either a specific predicate or predicates selected at random from a small domain, rather than using a truly random predicate (as in the foregoing presentation). The advantage of these suggestions is that the description of the predicate is shorter, and so one may use larger values of $\ell$. Two specific suggestions follow:

1. Use the predicate that partitions its input into two equal length strings and takes their inner product modulo 2 . That is, $P\left(z_{1}, \ldots, z_{2 t}\right)=\sum_{i=1}^{t} z_{i} z_{t+i} \bmod$ 2 .

In this case, the predicate is described without reference to $\ell$, and so any value of $\ell$ can be used (in practice). This suggestion is due to Adi Shamir.

2. Use a random low-degree $\ell$-variant polynomial as a predicate. Specifically, we think of a random $\ell$-variant polynomial of degree $d \in\{2,3\}$ over the finite field of two elements, and such a polynomial can be described by $\left(\begin{array}{l}\ell \\ d\end{array}\right)$ bits. In practice, even for $d=3$, we may use $\ell=32$ (since the description length in this case is less than $6 \mathrm{~K}$ bits).

On the other extreme, for sake of simplifying the analysis, one may use different predicates in each application (rather than using the same predicate in all applications).

\subsection{Directions for investigation}

1. The combinatorial properties of the function $f_{n}$. Here we refer to issues such as under what conditions is $f_{n}$ 1-to-1 or merely "looses little information"; that is, how is $f_{n}\left(X_{n}\right)$ distributed, when $X_{n}$ is uniformly selected in $\{0,1\}^{n}$. One can show that if the collection $\left(S_{1}, \ldots, S_{n}\right)$ is sufficiently expending (as defined above), then $f_{n}\left(X_{n}\right)$ has min-entropy $\Omega(n)$; i.e., $\operatorname{Pr}\left[f_{n}\left(X_{n}\right)=\alpha\right]<$ $2^{-\Omega(n)}$, for every $\alpha \in\{0,1\}^{n}$. We seek min-entropy bounds of the form $n-O(\log n)$. 
2. What happens when $f_{n}$ is iterated? Assuming that $f_{n}$ "looses little information", iterating it may make the inverting task even harder, as well as serves as a starting point for the next item. ${ }^{6}$

3. Modifying the construction to obtained a "keyed"-function with the hope that the result is a pseudorandom function (cf. [9]). The idea is to let the key specify the (random) predicate $P$. We stress that this modification is applied to the iterated function, not to the basic one. ${ }^{7}$ We suggest using $\Theta(\log n)$ iteration; in practice $3-5$ iterations should suffice.

Our construction is similar to a construction that was developed by Alekhnovich et. al. [3] in the context of proof complexity. Their results may be applicable to show that certain search method that are related to resolution will require exponential-time to invert our function [Avi Wigderson, private communication, $2000]{ }^{8}$

\subsection{Inspiration}

Our construction was inspired by the construction of Nisan and Wigderson [11]; however, we deviate from the latter work in two important aspects:

1. Nisan and Wigderson reduce the security of their construction to the hardness of the predicate in use. In our construction, the predicate is not complex at all (and our hope that the function is hard to invert can not arise from the complexity of the predicate). That is, we hope that the function is harder to invert than the predicate is to compute. ${ }^{9}$

2. The set system used by Nisan and Wigderson has different combinatorial properties than the systems used by us. Specifically, Nisan and Wigderson ask for small intersections of each pair of sets, whereas we seek expansion properties (of a type that cannot be satisfied by pairs of sets).

Our construction is also reminiscent of a sub-structure of of the DES; specifically, we refer to the mapping from 32-bit long strings to 32-bit long strings induced by the eight S-boxes. However, the connection within input bits and output bits is far more complex in our case. Specifically, in the DES, each of the 8 (4-bit) output strings is a function (computed by an S-box) of 6 (out of the 32) input

\footnotetext{
${ }^{6}$ An additional motivation for iterating $f_{n}$ is to increase the dependence of each output bit on the input bits. A dependency of each output bit on all output bits is considered by some researchers to be a requirement from a one-way function; we beg to differ.

7 We note that applying this idea to the original function will definitely fail. In that case, by using $2^{\ell}$ queries (and inspecting only one bit of the answers) we can easily retrieve the key $P$.

8 This conjecture was proved in [7]. We mention that in the original posting of this work we expressed the opinion that this direction requires further investigation.

${ }^{9}$ We comment that it is not clear whether the Nisan and Wigderson construction can be broken within time comparable to that of computing the predicate; their paper only shows that it cannot be broken substantially faster.
} 
bits. The corresponding 8 subsets have a very simple structure; the $i^{\text {th }}$ subset holds bit locations $\{4(i-1)+j: j=0, \ldots, 5\}$, where $i=1, \ldots, 8$ and 32 is identified with 0 . Indeed, inverting the mapping induced on 32 -bit strings is very easy. ${ }^{10} \mathrm{In}$ contrast, the complex relation between the input bits corresponding to certain output bits, in our case, defeat such a simple inversion attack. We stress that this complex (or rather expanding) property of the sets of input bits is the heart of our suggestion.

\section{Subsequent Work and Afterthoughts}

As evident from the Introduction (as well as from Section 4), our primary motivation for this proposal was to address a pratical concern. We hoped that the apparently low cost of a hardware implementation should make this proposal very appealing to practice. We further hoped that the simplicity of the proposal may encourage theoretically inclined researchers to study it, and that such research may generate more confidence in this proposal. From this perspective and for reasons outlined next, it felt preferrable to promote a setting of $\ell=O(\log n)$.

At the time, we were unclear as to what may be the best choice of a predicate $P$, and our feeling was that most predicates (or a random predicate) will do. Believing that it is best that $P$ lacks any structure (and thus using a random $P$ ), the truthtable of $P$ had to be hard-wired in the function (or appear as part of the input and output to the function). For that reason, $\ell=O(\log n)$ was an absolute limit. As clear from the text, we did not rule out the possibility of setting $\ell=O(1)$, but letting $\ell$ be as large as possible felt safer (cf. Footnote 1 ).

In contrast to our initial motivations, the complexity theory community becames more interested in the possibility of setting $\ell=O(1)$, as this yields a function in $\mathcal{N C}_{0}$. Interest in this aspect of the current work was fueled by the celebrated work of Applebaum, Ishai, and Kushilevitz [2], since one of their main results implies that $\mathcal{N C}_{0}$ contains one-way functions if and only if $\mathcal{N C}_{1}$ contains such functions. In fact, it is fair to say that the current work was practically rediscovered after [2].

Subsequent studies have shakened our confidence that a random predicate $P$ is the best possible choice for our proposal. In particular, generalizing our proposal to functions with $m=O(n)$ output bits (rather than $n$ output bits), Bogdanov and Qiao showed [6] that a necessary requirement for security is using a balanaced predicate $P$ (i.e., $P$ such that $\left|\left\{z \in\{0,1\}^{\ell}: P(z)=1\right\}\right|=2^{\ell-1}$ ). The use of balanaced predicates is also advocated in $[1,7]$.

\section{Acknowledgments}

We are grateful to Noga Alon, Adi Shamir, Luca Trevisan, and Avi Wigderson for useful discussions.

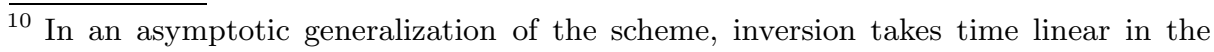
number of bits. 


\section{References}

1. B. Applebaum, B. Barak, and A. Wigderson. Public-key cryptography from different assumptions. In 42nd STOC, pages 171-180, 2010.

2. B. Applebaum, Y. Ishai, and E. Kushilevitz. Cryptography in NC0. SICOMP, Vol. 36 (4), pages 845-888, 2006.

3. M. Alekhnovich, E. Ben-Sasson, A. Razborov, and A. Wigderson. Pseudorandom Generators in Propositional Proof Complexity. In 41st FOCS, pages $43-53,2000$.

4. N. Alon. Eigenvalues, Geometric Expanders, Sorting in Rounds, and Ramsey Theory. Combinatorica, Vol. 6, pages 207-219, 1986.

5. N. Alon And V.D. Milman. $\lambda_{1}$, Isoperimetric Inequalities for Graphs and Superconcentrators, J. Combinatorial Theory, Ser. B, Vol. 38, pages 73-88, 1985.

6. A. Bogdanov And Y. Qiao. On the Security of Goldreich's One-Way Function. In 13th RANDOM, Springer, LNCS 5687, pages 392-405, 2009.

7. J. Cook, O. Etesami, R. Miller, and L. Trevisan. Goldreich's OneWay Function Candidate and Myopic Backtracking Algorithms. In 6th TCC, Springer, LNCS 5444, pages 521-538, 2009.

8. O. Gaber And Z. Galil. Explicit Constructions of Linear Size Superconcentrators. JCSS, Vol. 22, pages 407-420, 1981.

9. O. Goldreich, S. Goldwasser, and S. Micali. How to Construct Random Functions. JACM, Vol. 33, No. 4, pages 792-807, 1986.

10. A. Lubotzky, R. Phillips, P. Sarnak. Ramanujan Graphs. Combinatorica, Vol. 8, pages 261-277, 1988.

11. N. Nisan And A. Wigderson. Hardness vs Randomness. JCSS, Vol. 49, No. 2, pages 149-167, 1994. 